\title{
B-supergiants in IC1613: testing low-Z massive star physics and evolution
}

\author{
Inés Camacho ${ }^{1,2}$, Miriam García ${ }^{3}$, Miguel A. Urbaneja ${ }^{4}$ \\ and Artemio Herrero ${ }^{1,2}$ \\ ${ }^{1}$ Instituto de Astrofísica de Canarias, La Laguna, Tenerife, Spain \\ ${ }^{2}$ Departamento de Astrofísica, Universidad de La Laguna, La Laguna, Tenerife, Spain \\ ${ }^{3}$ Centro de Astrobiología CAB (CSIC/INTA), Madrid, Spain \\ ${ }^{4}$ Institute of Astro and Particle Physics, Universität Innsbruck, Innsbruck, Austria
}

\begin{abstract}
The physical processes taking place in massive stars during their life and death are highly dependent on the metallicity $(\mathrm{Z})$ of their parent cloud. Observations of these stars in low-Z nearby galaxies are crucial to understand these processes. IC1613 is the nearest Local Group galaxy with ongoing star formation and O-abundance lower than the SMC, although UV spectroscopy suggests it is not so metal poor. We performed a spectral analysis of early B-type stars in the galaxy, obtaining physical parameters and abundances. Our results confirm the low O-abundance of IC1613.
\end{abstract}

Keywords. IC1613, massive stars, galaxy, abundances, metallicity

We have studied 11 early B-type stars in IC1613. We performed high quality spectral analysis of the data, using a non-classical automatic analysis method (GPMC, Urbaneja in prep.) and an optimized grid of 800 FASTWIND models. We obtained 11 physical parameters and abundances simultaneously ( $\mathrm{T}_{\text {eff }}, \log , H e, \xi, \beta, \dot{M}, \mathrm{O}, \mathrm{N}, \mathrm{C}, \mathrm{Mg}$, and $\mathrm{Si}$ ). From this analysis, we draw three main conclusions: a) We recover an average O-abundance of $12+\log (\mathrm{O} / \mathrm{H})=7.86 \pm 0.12$, in agreement with other stellar and nebular studies in the galaxy (as shown in Fig. 1-left), and confirm the low O-abundance of IC1613. b) The derived $\mathrm{T}_{\text {eff }}$ of our supergiant sample are consistent with the Markova \&

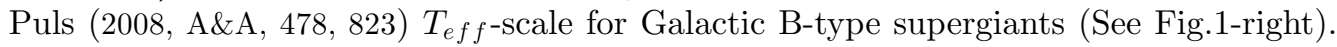
This rather suggests there is not a significant effect of metallicity on the effective temperature for B-supergiants. c) Comparing the resulting abundances with the predictions of evolutionary models (Brott et al., 2011, A\&A, 530, A115), the low metallicity seems to favor high initial rotational velocities, although present Vsini values are lower than predicted.
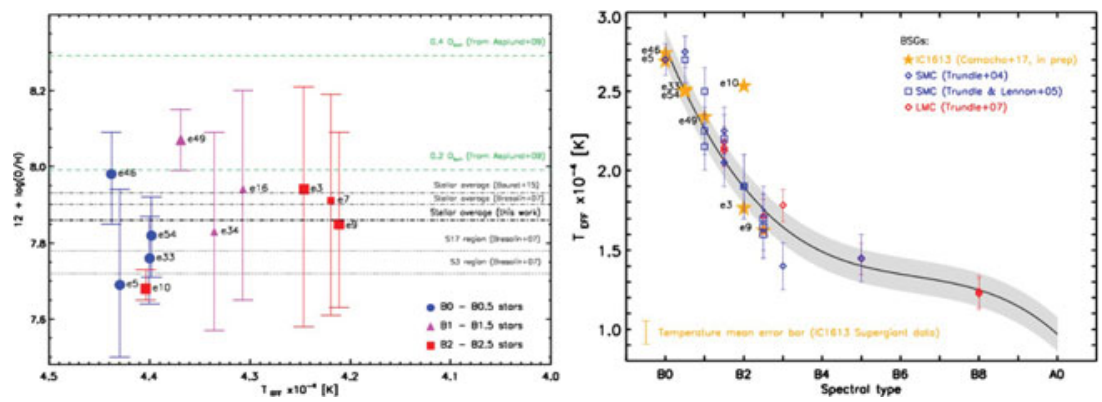

Figure 1. LEFT: Stellar O-abundances in IC1613 derived in this work. Other stellar and nebular studies are also included. RIGHT: Temperature scale for Galactic B-supergiants (B-SG) derived by Markova \& Puls, together with the B-SG $T_{\text {eff }}$ 's derived in this work (orange stars). 